ISSN: 0210-1696

DOI: http://dx.doi.org/10.14201/scero201546181106

\title{
CLAVES DE FUTURO EN LA ATENCIÓN Y APOYO A LAS PERSONAS CON DISCAPACIDAD INTELECTUAL Y DEL DESARROLLO. ¿QUÉ NOS DICE LA CIENCIA?
}

\section{Keys to Future in the Care and Support for People with Intellectual and Developmental Disabilities. What Tell us Science?}

Climent Giné Giné

Universitat Ramón Llull. Facultad de Psicología, Ciencias de la Educación y del Deporte. Blanquerna. Barcelona

Delfín Montero Centeno Universidad de Deusto. Facultad de Filosofía y Ciencias de la Educación. Departamento de Pedagogía Social y Diversidad. Bilbao

Miguel Ángel Verdugo Alonso

Universidad de Salamanca. Facultad de Psicología. Departamento de Personalidad, Evaluación $y$ Tratamiento Psicológicos. Salamanca

Pere Rueda Quitllet

Fundación Ampans. Barcelona

prueda@ampans.cat

Susanna VERT TAPIA

Dincat. Barcelona

Recepción: 14 de octubre de 2014

Fecha de aceptación definitiva: 1 de diciembre de 2014

Biblid. [0210-1696 (2015) vol. 46 (1), n. ${ }^{\circ}$ 253, enero-marzo; 81-106]

RESUMEN: Este artículo describe las aportaciones de un debate cuyo objeto principal era responder, por parte de tres expertos, a una serie de tópicos, con la mirada puesta en un futuro de los próximos diez años, tales como: la evolución de los conceptos de inteligencia y de conducta adaptativa; la evaluación diagnóstica de la discapacidad; la calidad 
de vida como fuente de información para evaluar las políticas, así como las prácticas de los servicios; las condiciones que debe tener un servicio para que sea realmente un servicio centrado en la persona; los servicios basados en la comunidad; las necesidades de apoyo de las familias; el papel de los padres; o bien la propia participación de las personas con discapacidad en el desarrollo de los servicios.

El debate se organizó en base a tres grandes bloques de contenido: elementos diagnósticos, constructos del campo de la discapacidad intelectual y prestación de apoyos.

Este artículo finaliza con un decálogo de conclusiones en base a las argumentaciones de los intervinientes.

Palabras Clave: personas con discapacidad intelectual; inteligencia; conducta adaptativa; calidad de vida; evaluación diagnóstica; autodeterminación; inclusión social; servicios centrados en la persona; familias; formación de profesionales.

ABSTRACT: This article describes the contributions of a round table whose main purpose was to respond, by three experts, a series of topics, with the look anytime in the next ten years, such as: the evolution of the concepts of intelligence and adaptive behavior; the diagnostic evaluation of disability; the quality of life as a source of information to evaluate the policies, as well as the practices of the services; the conditions that must have a service to make it really a service focused on the person; services based in the community; the needs of supporting families; the role of the parents; either the own participation of persons with disabilities in the development of services.

The discussion was organized on the basis of three large blocks of content: diagnostic elements, constructs in the field of intellectual disabilities, and provision of support.

This article ends with a Decalogue of conclusions based on the arguments of the parties.

KEY WORDS: people with intellectual disabilities; intelligence; adaptive behavior; quality of life; diagnostic evaluation; self-determination; social inclusion; services focused on the person; families; training of professionals.

\section{Introducción}

$\mathrm{E}$ N LOS PRÓXIMOS AÑOS TAL VEZ VEAMOS CAMBIOS IMPORTANTES en los modelos de prestación de servicios a las personas que presentan una Discapacidad Intelectual o del Desarrollo. Modelos basados en los apoyos comunitarios y en la coparticipación para la producción de servicios y programas por parte de los diferentes grupos de interés, incluida la sociedad más cercana.

Seguramente que estos cambios vendrán producidos no solo por el entorno socioeconómico, sino también por el estado del arte en el campo de la Discapacidad, por los resultados de la investigación, por la búsqueda de soluciones a las necesidades planteadas por las personas con discapacidad intelectual y del desarrollo y sus familias.

La Fundación AMPANS y DINCAT han organizado un debate, aprovechando la participación de los doctores: Climent Giné, Delfín Montero y Miguel Ángel Verdugo como miembros del jurado del Premio de Investigación e Innovación 
de Ampans, que pretende darnos claves de cómo la evidencia orienta el futuro. En la década del 2020, ¿cómo evolucionarán los conceptos de inteligencia, de conducta adaptativa?; la Clasificación Internacional de las Enfermedades (ICD-11), el Manual Diagnóstico y Estudio de los Trastornos Mentales (DSM-5) ¿’hacia dónde se dirigen y cómo confluyen?; ¿en qué medida las familias pueden tener nuevas concepciones respecto a los servicios, diferentes necesidades de apoyo, los próximos años?; ¿deberemos coger indicadores de desarrollo humano para establecer las políticas en el campo de la discapacidad intelectual y del desarrollo?

Éstos, y otros tópicos que han planteado los participantes, han sido objeto de análisis y de debate, a través de la metodología de entrevista en profundidad en base a preguntas que, en cierta medida, sustituyen a la metodología de los "focus grup", a los tres profesores universitarios que han dedicado, y siguen dedicando, sus años de estudio e investigación al área de la discapacidad intelectual y del desarrollo.

El resultado del debate da pie a poder sacar algunas conclusiones, que hemos convertido en un decálogo, que ha sido evaluado y validado por los propios profesores expertos.

Dr. Climent Giné, profesor emérito de la Facultad de Psicología, Ciencias de la Educación y del Deporte Blanquerna (Universidad Ramón Llull). Investigador principal del grupo de investigación "Discapacidad y Calidad de Vida: Aspectos Educativos”.

Dr. Delfín Montero, profesor titular del Departamento de Pedagogía Social y Diversidad de la Universidad de Deusto. Doctor en Discapacidad y Necesidades Educativas Especiales. Autor de diferentes libros y manuales sobre la Conducta Adaptativa.

Dr. Miguel Ángel Verdugo, catedrático de Psicología de la discapacidad en la Universidad de Salamanca. Doctor en Psicología. Director del INICO, así como del Máster en Integración de Personas con Discapacidad y de Siglo Cero. Autor de libros y director de investigaciones.

\section{El debate}

\section{A. Elementos diagnósticos}

Pere Rueda (P.): ¿Qué es la inteligencia boy? El actual concepto, en un punto de corte de CI <70 (70-84 "borderline”), ¿ hasta cuándo se mantendrá?, ¿qué cambios se vislumbran en el horizonte?

Delfín Montero (DM): Qué es la inteligencia es una de las grandes preguntas. Para responderla se podría encontrar un paralelismo en determinados panteones de religiones politeístas, en los que por encima de los dioses o diosas encargados de un determinado aspecto de la realidad hay un dios único que regula todo este panteón y que sería lo que llamamos el factor "g”. Después de décadas y décadas de investigaciones factoriales sobre la naturaleza de la inteligencia, todo parecería apuntar a que hay un factor, pero también es una inteligencia, al mismo tiempo, múltiple con diversos tipos de inteligencia. Esta concepción de un factor único es uno de los fundamentos 
por los cuales la AAIDD (Asociación Americana sobre la Discapacidad Intelectual y del Desarrollo) y la APA (Asociación Americana de Psiquiatría) han mantenido la inteligencia como factor general como uno de los criterios de la definición y la que le da a los tests de inteligencia el papel que aún tienen.

¿Hasta cuándo se mantendrá? No lo sé, pero mucho me temo que tenemos CI para rato. ¿Y por qué digo esto? Por puro pragmatismo; estamos ante una herramienta, como es el caso de los tests de inteligencia que funcionan fenomenalmente bien, es decir, desde un punto de vista técnico son uno de los grandes logros de la psicología contemporánea. Es evidente que detrás de ellos hay historias de escándalos, algunas de ellas dignas de una opereta, pero se trata de unas herramientas de primera magnitud que permiten estimar, de una manera rápida, válida y fiable, algo que tiene que ver con la inteligencia. La discusión está en si esto que estiman los tests de inteligencia es algo que podemos asimilar a una cosa tan grande como es la inteligencia. Antoni Castelló dice que los tests de inteligencia son buenos tests pero con un mal nombre, ya que probablemente no están midiendo toda la complejidad que hay detrás de la conducta humana inteligente. Esto es algo que salta a la vista. Detrás de constataciones así está la decisión por parte de la AAIDD de incluir la conducta adaptativa en 1959 como otro criterio de la discapacidad intelectual, como un indicador de un tipo de competencia relacionada con la solución de problemas de lo cotidiano, del que no da cuenta un cociente intelectual.

Es posible que podamos ver una evolución en algún momento. Puede que dentro de 10 o 20 años haya alguna iniciativa para rebajar el punto de corte, o flexibilizarlo, algo que ya ha hizo la AAMR (Asociación Americana sobre el Retraso Mental) en el año 92, cuando hablaba de un punto de corte de entre 70 o 75, algo que, por cierto, generó protestas mayúsculas, por ejemplo de la división de las discapacidades del desarrollo de la APA (American Psychological Association).

\section{P: Las propuestas de Greenspan sobre inteligencia social en lo que respecta a "inge-} nuidad", "creabilidad", ¿qué aportan?

DM: Greenspan es uno de los cerebros grises de la AAIDD. Me interesaron sobremanera sus trabajos a finales de los 80 , en el momento que descubro que habla de un modelo de competencia personal compuesto de inteligencia conceptual, inteligencia práctica e inteligencia social. Sus ideas influyeron mucho en la revolucionaria propuesta de la AAIDD del año 1992.

Si pensamos en el horizonte de los 2020, él ha hecho propuestas muy novedosas en las cuales se sugiere que se defina la discapacidad intelectual como una limitación significativa de las capacidades cognitivas a partir de los tres indicadores: la inteligencia conceptual, la inteligencia práctica y la inteligencia social; una propuesta muy interesante y directamente comprensible por el común de la ciudadanía; tal vez más que el concepto de conducta adaptativa, que hay que explicarlo siempre para los que no están en el campo.

Una de sus líneas de trabajo más recientes tiene que ver con las situaciones de credibilidad, inocencia, fruto de su capacidad reflexiva sobre la fragilidad y la vulnerabilidad de las personas con DI para ser engañadas; siendo éste un tema no solamente propio de la DI, sino que también puede aplicarse a las personas mayores; algo de 
repercusión potencialmente grande, por ejemplo, en sentencias de incapacidad. Y por lo que respecta a las personas con DI, un tema más en la agenda educativa del alumnado con DI, que con frecuencia no sabe reaccionar adecuadamente ante claves o situaciones en las que puede ser engañado. No podemos hacer unos buenos planes de transición a la vida adulta si no trabajamos estos aspectos en edades más tempranas; así como para manejarse por la comunidad con autonomía y seguridad.

Miguel Ángel Verdugo (MAV): Greenspan es el hombre de reflexión más profunda de la AAIDD en el tema de la Conducta Adaptativa por los aspectos de análisis y de dar valor al concepto de comportamiento adaptativo. Él dice: ¿ por qué hablamos tanto de inteligencia?, es el que plantea el modelo más radical de Conducta Adaptativa; y es el que plantea estos conceptos de ingenuidad, credibilidad de las preguntas. En sus análisis dice que las personas con DI se caracterizan o distinguen no por su inteligencia, sino por este carácter de vulnerabilidad de ser abusados por otros, o para ser manipulados o utilizados.

LAS PERSONAS CON DI SE CARACTERIZAN O DISTINGUEN NO POR SU INTELIGENCIA, SINO POR EL CARÁCTER DE VULNERABILIDAD DE SER ABUSADOS POR OTROS, O PARA SER MANIPULADOS O UTILIZADOS.

Greenspan es de gran importancia por sus implicaciones prácticas, así como por sus escritos y sus reflexiones, que son de gran valor para dar significado a la Conducta Adaptativa y para hacer este giro hacia comportamientos que no se tenían en cuenta a la hora de valorar o evaluar, cuando pensamos en términos de inclusión comunitaria de la persona.

Es una persona de referencia. Quizá los dos autores que, en este momento, son los mayores referentes son Greenspan y Schalock para entender el actual constructo, así como por la generación de implicaciones prácticas y de aplicación.

P: Ya que bemos empezado a bablar de inteligencia práctica, social, conceptual, el paralelismo entre Inteligencia social, conceptual y práctica, y la conducta adaptativa social, cognitiva y práctica. ¿Cómo irá evolucionando? $Y$ ¿ en qué medida el concepto de Conducta Adaptativa irá cogiendo terreno al de Inteligencia?

MAV: Hay que ver la evolución histórica. Lo que hemos hecho es: desde darle un valor, casi exclusivo, a la inteligencia para el diagnóstico, a empezar a introducir la conducta adaptativa. Ésta ha ido creciendo en importancia y las previsiones son que siga creciendo en importancia, aunque se siga manteniendo, en el diagnóstico de la discapacidad intelectual, la limitación significativa de la inteligencia, su alejamiento de la normalidad, en dos desviaciones típicas inferior a la media, es decir, un $3 \%$ de la población puede estar en ese criterio. 
En la última revisión de la definición de 2010 (2011 en castellano) ha llegado a plantearse la eliminación del término "discapacidad intelectual”, lo cual era muy arriesgado porque generaría más problemas. La conducta adaptativa tiene un mayor peso para discriminar el diagnóstico y para entender un poco el funcionamiento de la persona y esto tiene mayores implicaciones prácticas.

LA CONDUCTA ADAPTATIVA TIENE MÁS PESO QUE LA INTELIGENCIA PARA DISCRIMINAR EL DIAGNÓSTICO Y PARA ENTENDER UN POCO EL FUNCIONAMIENTO DE LA PERSONA

¿Qué previsión hay? Pues se volverá a discutir, probablemente, en perspectivas de 2018-20; y quienes, en ese momento, estén en la discusión, volverán a discutir qué peso tienen, en la discapacidad intelectual y en su definición, la inteligencia y la conducta adaptativa y, probablemente, la conducta adaptativa adquiera más peso. Tal vez lo psicométrico se reduzca al diagnóstico discriminativo, a un entendimiento del patrón general de comportamiento más académico. También puede haber la idea de seleccionar un único término: "competencia”, y subsumir ambos conceptos (intelectual y adaptativo) en uno solo. Lo que no casa tanto es hablar de inteligencia y la parte conceptual de la conducta adaptativa; esta parte conceptual habrá que ver qué comparte con inteligencia y qué comparte con conducta adaptativa.

Ahora mismo estamos dando un paso enorme, la publicación de la primera prueba que va a ser útil para el Diagnóstico, la “DABS”, la escala de diagnóstico de la conducta adaptativa, que ya está pendiente de publicarse en inglés este año 2014 o en 2015, y en castellano después. Será la primera escala construida para evaluar la triple vertiente de conducta adaptativa: conceptual, práctica y social; y que a la vez discrimina muy bien aspectos de ítems más o menos difíciles (está construida en base a la teoría de respuesta a los ítems). Esta prueba, al igual que otras, tendrá implicaciones importantes para la evaluación en ámbitos educativos.

DM: Hay bastantes cosas interesantes en lo que dice Miguel Ángel Verdugo, yo quiero destacar una para ligarlo con lo que decía antes. Me parece muy llamativo que la conducta adaptativa tenga este carácter tripartito: práctico, social y conceptual. Hay ahí aparentes incongruencias, la conducta adaptativa conceptual ¿no parece más vinculada con el otro polo de la definición?, el de la inteligencia. Bien pudiera ser que lleguemos dentro de unos años a la propuesta de Greenspan de la que hablaba antes: tal vez lo más sensato sería hablar de tres tipos de inteligencia para definir la discapacidad intelectual. Además, él lo plantea con una sencillez meridiana, si definimos discapacidad intelectual como un déficit entre estos tres tipos de inteligencia y hacemos equivaler la inteligencia conceptual a una dimensión que podemos medir con los test de inteligencia actualmente disponibles, si partimos además de la hipótesis de que las escalas de conducta adaptativa que tenemos ahora mismo miden, fundamentalmente, lo que podríamos denominar inteligencia práctica. Nos puede quedar la dimensión de inteligencia social sin una herramienta clara que la mida; aunque ahora mismo, con las escalas que van a aparecer en nuestro panorama, como es el caso de la "DABS" y el 
“ABAS-II”, ya empezamos a tener también, dentro de esas herramientas, unas dimensiones que pueden permitir tener algún indicador que podríamos denominar inteligencia social. Evidentemente todavía no hay una herramienta que sea satisfactoria para valorar la dimensión de inteligencia social, pero éstas pueden representar un avance.

P: Ya que estamos en el tema diagnóstico, ¿por dónde se encaminan los temas de evaluación diagnóstica de la discapacidad en los próximos 10 años?

Climent Giné (CG): En el campo de la DI, en los próximos años, tendremos que tener en cuenta dos aspectos fundamentales, aparte de un tercero, si consideramos la edad de aparición. Me refiero a (1) las limitaciones significativas en el funcionamiento intelectual; y (2) las limitaciones significativas en la conducta adaptativa. Debe recordarse que estos criterios ya se están utilizando desde los años 60 y 70.

¿Qué nos debe hacer suponer que esto cambiará en un futuro? Creo que poco cambiará en los próximos años; evidentemente, se va a profundizar en los tres componentes de inteligencia: conceptual, práctica y social, en cuanto a instrumentos de evaluación y mejora conceptual de los términos, pero en realidad los dos grandes criterios van a continuar.

EN LOS PRÓXIMOS AÑOS SE VA A PROFUNDIZAR EN LOS TRES COMPONENTES DE INTELIGENCIA: CONCEPTUAL, PRÁCTICA Y SOCIAL, EN CUANTO A INSTRUMENTOS DE EVALUACIÓN Y MEJORA CONCEPTUAL DE LOS TÉRMINOS

¿Qué nos va a deparar, pues, el futuro? El peso no va a estar tanto en el diagnóstico, sino en sus consecuencias para la intervención; probablemente el futuro nos va a generar investigación que intentará asociar los resultados del diagnóstico al sistema de apoyos. ¿Cómo reflejar el diagnóstico en objetivos y propuestas de trabajo? En este sentido, no se trata tanto de conocer qué "tiene", como procurar aquellas oportunidades, experiencias, que permitan una vida de calidad. La investigación va a priorizar estos temas.

EL FUTURO NOS VA A GENERAR INVESTIGACIÓN QUE INTENTARÁ ASOCIAR LOS RESULTADOS DEL DIAGNÓSTICO AL SISTEMA DE APOYOS

P: ¿Cómo medir la inteligencia social con indicadores verdaderamente fiables? (pregunta de Aprosub)

DM: Hay un cuerpo de teoría lo suficientemente amplio para trabajar en esta línea. Disponemos de una serie de herramientas que nos permiten tener estimaciones objetivas.

(C) Ediciones Universidad de Salamanca

Siglo Cero, vol. 46 (1), n. ${ }^{\circ} 253,2015$, enero-marzo, pp. 81-106 
Podemos realizar observaciones segmentadas en el tiempo, también tenemos tests y herramientas que permiten valorar los múltiples componentes de la inteligencia social, una competencia humana sumamente compleja, conformada, por ejemplo, por la comprensión de "las buenas costumbres", la capacidad de poder evaluar los conceptos éticos de la conducta, la capacidad de elaborar modelos de cómo funciona la persona, etc. Cualquier instrumento que pudiéramos desarrollar para medir todo esto, incluso, podría crearnos una cierta insatisfacción. Ahora bien, en este momento, como decía antes, ya tenemos puntos de partida, instrumentos que pueden darnos una serie de indicadores, por ejemplo, la DABS, donde hay una parte que evalúa las habilidades adaptativas de carácter social. De todas formas, el carácter descriptivo de estas herramientas acaba por hacernos echar en falta el componente cognitivo de, por ejemplo: ¿por qué lo hace?, ¿lo hace libremente?, ¿lo hace coaccionado?... Así pues, tenemos mimbres suficientes para poder empezar a trabajar en este campo, pero todavía hay mucho camino por recorrer.

CG: Tendríamos que plantear a los investigadores que están trabajando en estos temas un cierto esfuerzo y rigor en precisar lo que se entiende por integración social. En cuanto a las capacidades cognitivas se distingue entre la inteligencia conceptual, práctica y social; ¿dónde está el común denominador?, ¿cuáles son los límites entre una y otra? Si no tenemos claro lo que pensamos medir, nos va a ser difícil tener instrumentos; por lo que nos encontramos con instrumentos que no acaban de ser muy finos a la hora de evaluar el concepto.

P: Habría otra pregunta que cerraría este primer ciclo sobre elementos diagnósticos $y$ es este debate que comentaba yo antes de la Discapacidad Intelectual (ID) y el Desorden del Desarrollo Intelectual (IDD). ¿Qué podemos decir del debate abierto en si ID/IDD es una condición de salud o un problema funcional?

MAV: En la definición última de la AAIDD (2010) sobre DI planteamos establecer una relación directa con la Organización Mundial de la Salud y otros organismos para construir un modo de entender la discapacidad común con otras clasificaciones (ICD, DSM 5) para que no hubiera contradicciones, y hubo una influencia y una aceptación por parte de los equipos de la OMS para dar importancia a la conducta adaptativa, y eso es lo que ha reflejado el DSM 5.

Pero, en cierto modo, ellos se han tirado un poco a la piscina al reflejar los niveles de conducta adaptativa utilizando los términos ligero, moderado, severo y profundo, tradicionales de la inteligencia; y digo que se han tirado a la piscina porque no hay ninguna prueba o procedimiento que los pueda medir; por lo que al proponer estos niveles, lo que están haciendo es que detrás de esto, que tendría que ir una valoración con una base científica de tipo psicométrico de niveles diferenciados, vaya una valoración más bien de tipo clínico.

Evidentemente que han dado valor a lo que se les planteaba; han utilizado ya el término discapacidad intelectual, aunque mantienen, de manera simultánea, el del trastorno del desarrollo. Han aceptado utilizar el término Discapacidad Intelectual, 
han aceptado también el mayor valor de la conducta adaptativa y lo que hacen es dar un paso más allá, un cierto salto en el vacío, al incorporar los niveles y, en este sentido, no hay un procedimiento para ello.

La escala DABS nos dará un diagnóstico discriminativo de estas áreas de la conducta adaptativa, pero no nos va a permitir establecer niveles de ligero, severo y profundo, porque no está preparada, ni construida para ello; por lo tanto, habrá que resolver si es más factible o no, o sí que hay que desechar ese planteamiento. Eso solo es una pequeña contradicción dentro de la aceptación global que han hecho del uso del término y del valor del comportamiento adaptativo, más allá que el tradicional de inteligencia ligera, moderada o profunda.

CG: Brevemente y para complementar, al menos, uno de los aspectos que señalaba Miguel Ángel. Yo creo que hay que reconocer que el debate existe y disponemos de propuestas por ambas partes. Deberíamos tener presente que enfatizar que, fundamentalmente, es un problema de salud, o bien poner de relieve los aspectos funcionales, tiene ciertamente su importancia. Creo que obedece a modelos distintos, puesto que son organizaciones que vienen trabajando durante muchos años y están preocupados por estos temas desde sus propias concepciones; pero los modelos son los que están en la base de las propuestas.

La segunda consideración es el debate en torno a ¿cómo denominar este problema complejo que conocemos como discapacidad intelectual?, es un constructo al que, como hemos visto a lo largo de los años, se le han dado muchos nombres. Desde que empecé a estudiar estos temas he visto la preocupación por disponer de un "nombre" científicamente riguroso y políticamente correcto, pero al final ninguno acaba de consolidarse; cuando yo empecé a trabajar en una escuela especial, ésta se llamaba "para subnormales", y nadie se extrañaba. Hemos ido evolucionando; hoy en día unos ponen de relieve uno $u$ otro aspecto, y creo que es muy complicado, pero personalmente me inclino por entender que es un problema funcional. Evidentemente que hay aspectos que tienen que ver con lo orgánico, con el funcionamiento; pero la cuestión está en el análisis que podamos hacer; si enfatizamos los aspectos que tienen que ver con la salud, el déficit, o bien enfatizamos los aspectos que ponen en nuestras manos los modelos educativos. Me parece que es más un problema funcional y que hay que centrarse en los medios para poder trabajar.

DM: Una cosa más, para valorar lo que tiene de positivo la propuesta actual del DSM 5. Creo que hay una base científica para cuestionar que la Discapacidad Intelectual esté en el "Vademecum" de la psiquiatría actual. La discapacidad intelectual no es un problema psiquiátrico, ahora bien, todo esto, como en tantas cosas en nuestras vida privada y colectiva, tiene que ver con la historia. Hay determinadas cuestiones que nos acostumbramos a verlas en el paisaje y como siempre han estado ahí, y nos parece que forman parte del mismo; pero no tiene por qué ser así. Y lo mismo cabría decir con la Discapacidad Intelectual en la Clasificación Internacional de las Enfermedades; discapacidad intelectual no es una enfermedad; puede ir acompañada de, puede ser derivada de, pero no es una enfermedad. Lo que propone ahora mismo la APA 
(American Psychiatric Association), sobre los niveles de gravedad dentro de la discapacidad intelectual, utilizando tipologías de conductas adaptativas, recuerda muchísimo al manual de Grossman de la AAIDD del año 73. Es algo que ya estaba patentado.

LA DISCAPACIDAD INTELECTUAL NO ES UNA ENFERMEDAD; PUEDE IR ACOMPAÑADA DE, PUEDE SER DERIVADA DE, PERO NO ES UNA ENFERMEDAD

Ahora bien, también quiero decir, de todas formas, que es un progreso que la APA haya eliminado los subtipos de discapacidad intelectual; que los psiquiatras digan que la conducta adaptativa es importantísima para diseñar apoyos, que eso lo dice la versión actual del DSM 5, me parece que es un gran valor. Hay que verlo en plan positivo.

P: Vamos a terminar este bloque con una pregunta de un asistente: "¿Qué piensan ustedes del tema de la diversidad funcional derivada del modelo social de la discapacidad?”.

CG: Yo no quisiera ser poco respetuoso con la cuestión, pero entiendo que recurrir al término "diversidad funcional" responde a la necesidad de encontrar expresiones que se ajusten al modelo mental que se tenga del problema. A mi modo de ver, no define lo que se debe definir y, por tanto, lo veo como innecesario.

DM: Prácticamente de acuerdo con Climent Giné, porque siempre que veo a personas utilizando un término, para referirse a un amplio conjunto de personas dentro de ese gran paraguas llamado discapacidad, acaban hablando de diversidad funcional; diversidad funcional debida a problemas cognitivos, diversidad funcional debida a problemas de la comunicación, creo que estamos “mareando la perdiz”.

Entiendo, de todas formas, este afán de emancipación y de eliminar estigmas; y esto ha sido una constante también dentro del campo de la discapacidad; estamos quemando siempre la nave anterior porque ya se nos ha quedado muy obsoleta y parece que todo aquel que va montado en la nave se contagia de algo, y este es un pulso que no se acaba.

\section{B. Constructos del campo de la DI}

P: Pues pasamos al siguiente bloque, el bloque 2 que es el referido a los constructos de la discapacidad intelectual. Primero, una pregunta que lanzábamos sobre el tema de calidad de vida. Los datos de Calidad de Vida de cada persona, agregados, ¿son la mejor fuente de información para evaluar las políticas y prácticas de un servicio? 
MAV: Hay que ver cómo ha evolucionado el procedimiento del constructo. Lo que hace la Calidad de Vida es dar una gran importancia a los resultados personales. Nuestro trabajo es ayudar a la población y esto es lo que entendemos por resultados personales multidimensionales.

LO QUE HACE LA CALIDAD DE VIDA ES DAR UNA GRAN IMPORTANCIA A LOS RESULTADOS PERSONALES

Inicialmente hemos hecho mucho énfasis en las prácticas profesionales; como motor de cambio y de una nueva práctica evaluativa de generación de apoyos. El concepto de $\mathrm{CdV}$ ha evolucionado hacia un esquema integrador de la información evaluativa, diversa y adaptativa de apoyos, de planificación de apoyos. Pero en un momento determinado, la reflexión sobre el uso, y sobre las experiencias de aplicación en distintos países, lo que nos ha dado es que no puedes generar un cambio real en las prácticas profesionales, sobre todo generalizarlo, si no modificas la forma de entender las organizaciones, así como las políticas públicas.

Pero, sobre todo, lo que se plantea es un cambio organizacional, como puede leerse en el libro publicado por Schalock y por mí en el año 2010; y es que las organizaciones tienen que funcionar para generar resultados personales, pero también tienen que preocuparse por un crecimiento organizacional que mejore sus procesos internos, y hagan un análisis de la relación que existe entre la inversión económica y los resultados personales.

\section{LAS ORGANIZACIONES TIENEN QUE FUNCIONAR PARA GENERAR RESULTADOS PERSONALES}

La calidad de vida se entiende también como un modelo de cambio organizacional que integra los esfuerzos de cambio provenientes de distintos ámbitos, PCP, paradigma de apoyos, autodeterminación de las personas. Entonces, en política pública ¿qué es lo que ocurre?: un tema clave es que debe haber una alineación, una sinergia, entre lo que hacemos los profesionales, lo que hacen las organizaciones y el por dónde va la política pública, y que, cuando surge una contradicción entre éstos, se generan muchas barreras para poder aplicar modelos inclusivos. Si la Administración solamente te financia una plaza y un servicio cerrado, y no te financia por una actividad comunitaria, integradora, inclusiva, de prestación de apoyos, con profesionales haciendo tareas y roles diferenciados, entonces estamos en una contradicción que paraliza en lugar de ayudar.

Así pues, la política pública evalúa, también, los resultados personales, como uno de los criterios claves, no el único, a la vez que debe evaluar en qué medida se van proporcionando, de acuerdo con la Convención de las Naciones Unidas, más oportunidades, más tipos de servicios inclusivos, más conexiones entre la normalidad y la 
discapacidad; y en ese sentido digamos que la Calidad de Vida es algo que el administrador tiene que tener presente, tener en la cabeza, de cara generar un planteamiento novedoso sobre innovación, apoyando las innovaciones de las organizaciones con recursos económicos, diferenciando sus apoyos a las organizaciones en función de que haya un mayor compromiso o mejores resultados, al menos en mejorar los sistemas de funcionamiento.

Esto, las asociaciones lo tienen muy claro y hay muchos ejemplos en España y en Catalunya de trabajo bien hecho y de modos de cambio, procesos de cambio largos y de transformación.

Es preciso disponer de un modelo más definido de políticas públicas; pero lo que realmente pueden cambiar las cosas son las organizaciones, pero lo que hagan debe estar orientado con las políticas públicas. Al final, debe de haber un alineamiento de las políticas públicas con lo que hacen las organizaciones.

ES PRECISO DISPONER DE UN MODELO MÁS DEFINIDO DE POLÍTICAS PÚBLICAS QUE TENGA EN CUENTA LOS RESULTADOS PERSONALES

En definitiva, la responsabilidad pública es que da un dinero a personas que necesitan una compensación diferente a los demás en dedicación; y lo que tenemos que decir es si lo estamos logrando o no; sí, o no, a tal y cual persona. Y los datos agregados no dejan de ser una suma de lo que una organización tiene para reflexionar sobre qué cambios hacer o cómo reorientar los proyectos, es decir, sobre lo que está haciendo.

LOS DATOS AGREGADOS NO DEJAN DE SER UNA SUMA DE LO QUE UNA ORGANIZACIÓN TIENE PARA REFLEXIONAR SOBRE QUÉ CAMBIOS HACER O CÓMO REORIENTAR LOS PROYECTOS

CG: Desde mi punto de vista, y coincido con Miguel Ángel, probablemente una de las aportaciones más destacadas de los últimos años es llamar la atención de la sociedad, de los investigadores, pero también de los profesionales y de las administraciones que aquello que importa son los resultados personales que podamos obtener en términos de mejora de calidad de vida.

LO QUE DEBE IMPORTAR A LAS ORGANIZACIONES Y A LAS ADMINISTRACIONES PÚBLICAS SON LOS RESULTADOS PERSONALES QUE PODAMOS OBTENER EN TÉRMINOS DE MEJORA DE CALIDAD DE VIDA

Este es un tema que merecería mucha atención por parte de todos. Desde esta perspectiva, y centrándonos no en la literalidad de la pregunta, de si podemos utilizar 
datos agregados como fuente de información para evaluar las políticas y las prácticas, diría que los datos agregados de cada persona pueden servirnos, fundamentalmente, para promover el desarrollo y para evaluar si realmente con la práctica que estamos desarrollando obtenemos unos resultados más positivos. Los resultados personales los podemos entender también desde una perspectiva ecológica, a nivel más individual, a nivel de las instituciones organizativas y también desde las políticas. La perspectiva es que los datos que se tienen sobre una persona, evidentemente, pueden y deben servir para centrarlos y focalizarlos, desde el punto de vista de la persona, para promover el desarrollo y para evaluar los progresos; y evidentemente los datos agregados de una población pueden servirnos para las políticas porque son distintos niveles de utilización y análisis de la realidad. Es decir, volviendo a la perspectiva ecológica, qué mejor manera de reivindicar mejoras de calidad de vida sino a través de las políticas, a través de lo que es más propio del macrosistema: políticas, creencias.

P: Siguiendo el tema de calidad de vida últimamente leyendo algún artículo de Brown, o bien de Eric Emerson, nos hablan del término "estructura de capacidades". La "Estructura de Capacidades (o Aptitudes)" ("Capabilities"), ¿qué es?; ¿es una forma de pensamiento alternativa que puede ayudarnos a complementar la aplicación de la Calidad de Vida?

E incluso lo complementaría con otra pregunta, ¿en qué medida podríamos coger indicadores de desarrollo bumano para establecer políticas en el campo de la DID?

MAV: La calidad de vida se ha convertido en un concepto que integra todos los otros avances. Las dimensiones de la calidad de vida, entendemos, son el mejor sistema para integrar la información diagnóstica, evaluativa, de apoyos, de diferentes profesionales, o de conocimiento actitudinal, o de competencia inclusiva adaptativa. Cuando decimos integrada, el problema que tenemos no es la falta de información, ya que tenemos mucha información, y muy especializada, pues podemos tener informes de un logopeda, de un habilitador físico, de un psicólogo, de un educador, de monitores, sino que el problema está que, con todo esto, hacemos un esquema práctico de trabajo, accesible y práctico, que te permita organizar la atención en base a las dimensiones, que te permita organizar en esas áreas, esa información con la que los equipos discutan y prioricen la información inmediata, y la responsabilidad que pueden coger los profesionales cercanos sobre esos objetivos que se plantean; es la mayor virtud en ese sentido, tanto la actitudinal como de otros aspectos de avance que vaya a tener la discapacidad, que permite tener la información en una ventanita, digamos de cada dimensión, y el equipo decida a qué le da más prioridad; y dentro de esa ventana también están los sueños y preferencias de la persona, el proyecto de vida, los deseos; todo eso lo tienes que manejar para gestionar, qué objetivos concretos inmediatos en este trimestre son factibles de hacer para avanzar en la calidad de vida; este es un poquito el esquema. 

PARA INTEGRAR LA INFORMACIÓN DIAGNÓSTICA, EVALUATIVA, DE APOYOS, DE DIFERENTES PROFESIONALES

Creo que la mayor aportación de un enfoque centrado en la Calidad de Vida es la de integrar globalmente toda la información de evaluación sobre la persona, para priorizar y definir los objetivos. Y después te orienta cómo generar un esquema de apoyos para planificar y evaluar mejoras personales en la calidad de vida, todo ello de acuerdo con tu organización y su sistema de apoyos, de los profesionales y de los recursos naturales.

P: Entrariamos abora dentro de la dimensión autodeterminación. Me parece que, a veces, hay una cierta confusión en utilizar, casi como términos sinónimos, la autodeterminación y el control personal. Asimismo, si nos referimos a sistemas dirigidos al consumidor o sistemas de servicios controlados. ¿Qué áreas de su vida quieren controlar las personas con DID? A esta pregunta se añadiría otra para averiguar ¿hacia dónde se dirigirá la próxima investigación sobre autodeterminación?

MAV: La autodeterminación en sí tiene importancia porque es un constructo psicológico muy importante, que piensa en el individuo, aparte de ser una dimensión clara de la calidad de vida, una de las más altas que se relaciona con resultados de Calidad de Vida; luego, en sí, es un constructo y un principio de actuación inclusivo, en el sentido de dar valor a la persona y hacer que su participación sea activa, por ejemplo, ¿cómo definimos estrategias para mejorar la Calidad de Vida? La primera de ellas es activar la participación parcial del usuario, que tiene que ver con la autodeterminación, es una de las dificultades mayores; ¿y qué ocurre?, que la autodeterminación, en nuestra población, muchas veces tenemos que ayudarla a construir, cuanto más bajo es el nivel, objetivos propios, preferencias, a tomar decisiones y a tener oportunidades de elección, que es lo que tiene que ver con el constructo. Ellos tienen un recorrido personal importante en el constructo de autodeterminación, sobre todo en el ámbito de secundaria y de transición a la vida adulta que es, en toda España, el más débil; y por mucho que llevemos 15, 20 años hablando de ello todavía las experiencias son muy escasas; y son muy escasas las que están promoviendo planes activos de empoderar (empoderamiento). La autodeterminación también tiene que ver con la PCP; tiene que ver con todos esos aspectos que hemos señalado; en ese sentido, la autodeterminación tiene también su propio recorrido de avance en la persona; y también tenemos barreras importantes; a veces cuesta que la persona que tiene un bajo nivel sea activa en sus procesos de decisión y elección. Pero esto se ha de promover desde la educación para poder avanzar. 

INDIVIDUO, APARTE DE SER UNA DIMENSIÓN CLARA DE LA CALIDAD DE VIDA

Respecto a la investigación en autodeterminación, decir que se ha trabajado mucho en lo que afecta a la población con niveles de límite, ligero, medio, y mucho menos en población más afectada; si bien para esta población nos encontramos con estrategias y técnicas de participación parcial, de gestión de nuevas oportunidades.

Actualmente estamos trabajando en mejorar los aspectos evaluativos de la autodeterminación. En este sentido, el INICO, en este año 2014, hará disponible en su web la primera escala de evaluación de la autodeterminación con validación psicométrica apropiada para ser utilizada en nuestro contexto (Escala ARC-INICO). Esta escala se desarrolla a partir de la escala de Whemeyer, pero ha requerido cambios importantes (más de cinco años de trabajo) para tener éxito en la adaptación. La escala ARCINICO podrá ser utilizada, sobre todo, para adolescentes con discapacidad (de 16 años en adelante).

En USA se está trabajando en una nueva escala que sirva para la población con y sin discapacidad; de igual manera como se ha hecho con la conducta adaptativa; y ver cómo discrimina los ítems para personas con y sin discapacidad; y con ello, o a partir de ahí, poder basar, o incidir, los programas de apoyo. En los próximos años la investigación se orientará en aprender cómo se promueve la autodeterminación en la práctica y en los currículums que la facilitan. En España, en FEAPS, se dispone de documentos sobre autodeterminación de gran utilidad y que reflejan una experiencia rica, pero falta desarrollar muchas más experiencias aplicadas que se basen en resultados y datos.

P: ¿Qué soportes les hemos de dar a los jóvenes con DID para que sean más autodeterminados?

CG: Tal como señalaba MA al afirmar que la autodeterminación, por sí misma, es un constructo muy potente y uno de los elementos que más caracterizan y promueven la percepción de Calidad de Vida, deberíamos reconocer que en algún trabajo de investigación realizado en las personas con DI se encuentra que la autodeterminación es uno de los aspectos en el que las personas se muestran más necesitadas; los esfuerzos que se hacen no se corresponden con la importancia del tema en cuestión. DE VIDA EN LA QUE LAS PERSONAS SE MUESTRAN MÁS NECESITADAS 
Retomando la pregunta anterior de hacia dónde se va en la investigación; la persona de referencia viene siendo, a lo largo de estos últimos años, Whemeyer, pero, últimamente, una investigadora más joven, Karrie Shogren, de la Universidad de Kansas, acaba de publicar en Intellectual and Developmental Disabilities, del año 2013, un trabajo que me parece muy innovador, en el que observa que estamos acostumbrados a trabajar el tema de la autodeterminación como muy vinculado a objetivos formativos asociados al currículum y a las prácticas, a objetivos de transición a la vida adulta...; pero ella lo que nos propone, sin renunciar a trabajar también estos aspectos, es dotarlo de una perspectiva más amplia y, en este sentido, propone una perspectiva socioecológica que permita comprender, o que se orienta a comprender, los factores que, potencialmente, pueden impactar en la conducta autodeterminada; y entonces describe aquellos aspectos del microsistema, del mesosistema y del macrosistema que pueden tener un impacto en la autodeterminación de los jóvenes y, en este sentido, se pregunta por cuáles serían los apoyos; en su opinión, los apoyos serían aquellos que realmente pueden potenciar, en el microsistema, las oportunidades en la familia, por ejemplo, las rutinas diarias en casa a medida en que van creciendo; no podemos empezar a trabajar la autodeterminación cuando uno ya es adolescente, a los 12-14 años; de alguna manera se trata de incrustar las tareas, experiencias y objetivos de autodeterminación ya en el microsistema. Y ella también habla de cómo promover, en el microsistema, las redes sociales.

En el mesosistema, concretamente, sería cómo proveer de los apoyos que necesitan los jóvenes para promover experiencias y oportunidades, para expresar y para promover las habilidades de autodeterminación, y aquí sí que cita el sistema escolar y la propia comunidad; por lo tanto, que los adolescentes y los jóvenes puedan disponer de apoyos para tomar decisiones acerca de los servicios de la comunidad es una fuente de tener oportunidades, así como de recabar apoyos; $y$, finalmente, nos dice que las cuestiones de autodeterminación no pueden dejarse solo al diálogo entre el maestro o profesional y el individuo, sino que todos los aspectos del macrosistema que tienen que ver con las expectativas de las personas respecto a sus posibilidades de actuación, vinculadas a las creencias, son importantes. Por lo tanto, todo lo que podamos hacer que afecta a los valores, a las creencias, a las políticas tiene un impacto positivo en la promoción de la conducta autodeterminada.

QUE LOS ADOLESCENTES Y LOS JÓVENES CON DID DISPONGAN DE APOYOS PARA TOMAR DECISIONES ACERCA DE LOS SERVICIOS DE LA COMUNIDAD ES UNA FUENTE DE TENER OPORTUNIDADES Y DE RECABAR APOYOS

TODO LO QUE PODAMOS HACER QUE AFECTE A LOS VALORES, A LAS CREENCIAS, A LAS POLÍTICAS TENDRÁ UN IMPACTO POSITIVO EN LA PROMOCIÓN DE LA CONDUCTA AUTODETERMINADA 
Así pues, la investigación, creo que está haciendo este deslizamiento desde los aspectos más vinculados al currículum (a la persona, al contexto escolar), hacia una visión más ecológica que compromete los distintos valores.

DM: Hablando del tema de instrumentación, una línea prometedora es la que tiene que ver con la de las escalas de resultados personales; si bien hay relativamente poco publicado, sí que hay una base de trabajo realizado por los autores que llevaron a cabo la instrumentalización de la SIS (Escala de Intensidad de Apoyos) conjuntamente con un grupo de investigadores holandeses.

Y como decía CG de los aspectos de credulidad y demás, de que los aspectos de autodeterminación hay que empezarlos a trabajar desde bien temprano, desde la escuela, y, muy probablemente, desde una línea transversal a desarrollar para todo el currículum de los alumnos con trastornos del desarrollo, sea ésta de la autodeterminación, de enseñar estrategias de supervivencia en la calle, aspectos en los que estoy completamente de acuerdo. Y quiero ir más lejos aún, haciéndome una pregunta: ¿para cuándo vamos a tener unos materiales adaptados para profesores? En los que un profesor ordinario, sin necesidad de acudir a seminarios o de hacer una formación específica para trabajar con personas con DI, pueda acceder a las cuestiones clave con las que, como docente, tenga que enfrentarse para enseñar a un alumno con DI en estas cuestiones, algo que hacer, para otros, en el currículum ordinario, en todo lo que tiene que ver con el aprendizaje de ser ciudadano. Considero que hay soporte más que de sobra, no se trata de añadir nada nuevo, sino de desarrollar lo que ya existe; y, además, sería algo que beneficiaría a todo el alumnado enseñar cómo se toman decisiones de manera racional dentro de los grupos (por ejemplo, tema de las votaciones, las habilidades sociales...).

P: Para finalizar este bloque de autodeterminación, la autodeterminación ¿tiene una significación similar para las personas con DID que para sus familias?, ¿o significan cosas diferentes?

DM: Voy a contestar como observador participante, con algo que se lee, con relativa frecuencia, las conclusiones a las que llegan distintos grupos de autogestores. Tengo la sensación de que siempre hay un desfase entre lo que opinan los hijos y lo que opinan los padres, tengan o no DI; aunque al tratarse de un colectivo que tiene bastante más restringida su accesibilidad a un conjunto de servicios y posibilidades a los que tiene la población sin discapacidad, este hecho se hace más evidente.

Ojalá que sus elecciones puedan encontrar el apoyo necesario, si es preciso, como dicen las personas con discapacidad intelectual de "People First Central England" en su página web, y también el derecho a correr los mismos riesgos que cualquier otro, con apoyos si es preciso.

\section{Dos grandes cuestiones de futuro}

P: ¿Cuál será la perspectiva de los miembros de la Comunidad hacia la inclusión de las personas con DID? 
¿Cómo podemos dar apoyo al "rol” incrementado de la inclusión comunitaria?, es decir, ¿qué enfoques de intervención serán los más efectivos?

CG: Quisiera llamar la atención sobre un aspecto. Debemos tener en cuenta la perspectiva de los miembros de la comunidad que depende de los modelos mentales, de las creencias, de los valores; así pues, de algo que está en la propia sociedad, en el propio macrosistema. Por lo tanto, la pregunta sería: ¿cómo podemos modificar estos patrones, cómo podemos modificar los valores, las creencias de la sociedad? Porque es el caballo de batalla que nos va a permitir que cambie la mirada; y se ofrezcan mayores oportunidades a las personas con DI. Y desde este punto de vista lo que debo decir es que las experiencias de socialización son determinantes; la oportunidad que tienen las personas de poder convivir, de poder valorar y apreciar a las personas con DI es la única forma para poder promover este cambio deseable.

Además, evidentemente que al mirar la TV, así como otras actividades en las que las personas que presentan una DI cumplen determinados "roles", también ayuda. Pero la escuela es el instrumento fundamental que nos dota la sociedad para socializarles; ahora bien, lo cierto es que la convivencia, la presencia, pasan a ser un elemento inexcusable.

Así pues, lo que deberíamos hacer es promover una manera distinta de entender, una forma distinta de ver, a las personas con DI, que ponga de relieve sus capacidades y no sus dificultades. Para ello debemos servirnos de todo cuanto esté a nuestro alcance y en particular los medios de comunicación y las redes sociales.

DM: Yo quisiera hacer una reflexión más a nivel “macro”, como ciudadano. Hablar de inclusión social es hablar de un tema que tiene una gran carga política. Y me pregunto: ¿cuándo vamos a hablar de esto?, ¿qué hay de esto? , ¿para quién damos el voto?; igual, de repente, se empiezan a abrir determinadas cosas.

\section{Pregunta externa}

¿Quién tiene que convencer a las Administraciones Públicas que deben financiar servicios más inclusivos?

DM: Las propias personas con DI y sus representantes; los amigos; nosotros mismos.

CG: El problema es que en nuestra legislación la inclusión está muy presente, pero en la realidad está muy distante.

EN NUESTRA LEGISLACIÓN LA INCLUSIÓN ESTÁ MUY PRESENTE, PERO EN LA REALIDAD ESTÁ MUY DISTANTE

Que los servicios, desde la escuela hasta otro tipo de servicios que existan en la comunidad, sean realmente inclusivos, depende del compromiso de todos reflejado en 
el trabajo del "día a día”; no solo depende de recursos, aunque no sean desdeñables, sino de la voluntad de todos. Sin duda el compromiso de la Administración debería ir más allá; pero es una cuestión de prioridades; y debemos reconocer que estamos en un sector "perdedor" o, por decirlo de otra manera, de que les “importa poco"; las personas con DI son una minoría; una minoría que va luchando con pocos logros. $\mathrm{Ha}$ habido avances significativos, pero queda un largo trecho por recorrer. En este tema, nos encontramos con que los discursos son grandilocuentes, pero la realidad queda muy lejos.

\section{Prestación de apoyos}

P: ¿En qué medida las familias pueden tener nuevas concepciones respecto a los servicios, diferentes necesidades de apoyo, los próximos años?

¿Cuál será el papel de los padres en el futuro?

CG: En relación a la primera cuestión, creo que la familia puede tener nuevas concepciones respecto a los servicios; y el cambio va a ser posible en la medida que se avance hacia un modelo distinto de relación entre los profesionales y las propias familias; quiero decir, que se avance hacia un modelo de colaboración y no de "usuario".

Cuando los servicios que las organizaciones ofrecen a las familias están muy enraizados en el modelo de expertos, en el que nosotros les decimos lo que tienen que hacer, esto limita la posibilidad de participación de las familias, asignándoles un papel de meros receptores de nuestras orientaciones; además, muchas familias se sienten satisfechas con este papel.

CUANDO LOS SERVICIOS QUE LAS ORGANIZACIONES OFRECEN A LAS FAMILIAS ESTÁN MUY ENRAIZADOS EN EL MODELO DE EXPERTOS, SE LIMITA LA PARTICIPACIÓN DE LAS FAMILIAS, ASIGNÁNDOLES UN PAPEL DE MEROS RECEPTORES DE SUS ORIENTACIONES

Cambiar sus perspectivas y sus aspiraciones respecto a los servicios resulta complejo y va a ser posible en la medida que avancemos hacia un modelo de colaboración que les va a ayudar a cambiar su visión del servicio; que les va a ayudar a entender mejor un papel protagonista; y a expresar sus necesidades de apoyo.

Por lo tanto, el papel de los padres, de las familias, pasará a ser más activo, más comprometido; un rol en el que se reconozca también su conocimiento y su posible participación en la dirección u orientación que tomen los servicios y los apoyos que estén previstos para sus hijos o para sí mismos. Así pues, transitar del modelo de experto que condiciona la participación de las familias a unas determinadas prácticas, a un modelo de colaboración en el que se reconozcan sus aportaciones, evidentemente, poco a poco, generará en las familias nuevas expectativas. 
P: ¿Qué nos dice la investigación sobre las necesidades prioritarias de las familias?

CG: Respecto a las necesidades prioritarias que expresan las familias, quisiera decir, brevemente, algunos resultados de investigaciones llevadas a cabo por nuestro grupo de investigación:

1) Todo lo que tiene que ver con la información y la orientación. La información tiene que ser completa, veraz, puntual y creíble. La información es un componente importante de la calidad de vida familiar, y en este sentido se revela como una de las necesidades manifestadas por las familias.

2) Disponer de tiempo para sus propios proyectos personales; los padres demandan, cada vez más, que la presencia de la discapacidad no suponga una limitación. De hecho reconocen que a menudo lo es, para llevar a cabo sus proyectos personales, para poder dedicarse a las cosas que les puedan interesar, personal y colectivamente, para cumplir sus sueños y, por lo tanto, los canguros especializados y otras formas de apoyo adquieren un relieve importante.

3) Gestionar la conciliación; muchas familias manifiestan haber tenido que hacer renuncias importantes; $y$, si me permiten, muchas más en las madres que en los padres, para poder atender, sobre todo en las edades más tempranas, a sus hijos con discapacidad.

4) Hacer frente a determinados gastos, extras, que supone el cuidado de los hijos con discapacidad.

P: ¿Qué significa realmente "el apoyo a la familia”?

DM: ¿Cómo debe ser el apoyo a las familias? Como nota de humor lo resumiría en tres palabras: salud, dinero y amor.

P: ¿Qué necesita un Servicio, una organización, para avanzar hacia un Servicio Centrado en la Persona? Y viendo la perspectiva actual, ¿cuáles son las claves para que los servicios se orienten a la comunidad?

¿Qué indicadores principales podrían ser sistemáticamente utilizados para orientar las decisiones a nivel político en relación a las personas con DID? (CCAA, Estado). Indicadores de evidencia.

MAV: El cambio organizacional requiere un primer paso de cambio de modelo mental, digamos que se inicia conceptualmente. El entorno del mundo FEAPS ya lo sabe; sabe que hay nuevos modelos de gestionar y de trabajar. Pero hacer una transformación organizacional, el paso de lo conceptual a la práctica, lleva, como mínimo, entre 6 y 8 años. 
Pasar de un modelo de servicios a un modelo de apoyos; pasar de un modelo de servicios a uno que se base más en la utilización de los recursos comunitarios, en la participación de la persona, más allá, o fuera, de los que somos "especialistas" o profesionales, es un proceso que hay que planificar, avanzar con seguridad, agrupar a las organizaciones en este proceso de cambio, y esto requiere unas primeras fases de sensibilización y de formación; luego una planificación clara y un análisis autocrítico de cómo se están obteniendo los resultados.

Hay algunos ejemplos, todavía no muchos, pero que luego deben pasar al intercambio de experiencias para así poder dar pasos hacia adelante. Cada vez que avanzamos descubrimos mucho más las cosas que hacemos mal. Las mejores organizaciones que están trabajando el cambio organizacional son las que van descubriendo las mayores carencias y las mayores necesidades de cambio. Y ésta es la tónica en toda Europa, en el sentido de que el modelo de Calidad de Vida está sirviendo de revulsivo y modelo, orientando el cambio organizacional.

EL MODELO DE CALIDAD DE VIDA ESTÁ SIRVIENDO DE REVULSIVO Y DE MODELO, ORIENTANDO EL CAMBIO ORGANIZACIONAL

Actualmente, todos estamos en la búsqueda de un modelo basado en los Derechos, basado en la Comunidad; y éste es un proceso costoso y a partir del cual hay que hacer un cambio organizacional importante; y es la propia organización quien debe decidir cuál es el modelo organizacional más adecuado para lograr esto.

ESTAMOS EN LA BÚSQUEDA DE UN MODELO BASADO EN LOS DERECHOS, BASADO EN LA COMUNIDAD

Una de las cosas que tenemos clara es que debemos potenciar liderazgos más participativos, debiendo delegar responsabilidades de liderazgo.

En el libro publicado con Schalock sobre el Cambio Organizacional sugerimos algunas estrategias de cambio, pero luego hay que generar una cultura de aprendizaje en, y entre, todas las organizaciones, en la red; y luego alimentar el proceso de cambio de cara a dar pasos significativos.

La primera Escala que se va a hacer pública ya en 2014 en varios idiomas (castellano, inglés, holandés y chino) para orientar las necesidades de cambio organizacional es la Escala de Evaluación de la Eficacia y Eficiencia Organizacional (ver página web 
del INICO), escala que se basa en el modelo de CdV, y que consta de 20 indicadores. Ha sido elaborada por varias organizaciones de diferentes países, y en esa elaboración han participado gerentes y otros profesionales de las organizaciones.

Estos indicadores, de alguna manera, también pueden servir de evaluación de resultados de las políticas públicas.

¿Con qué indicadores cuenta la escala?; éstos están orientados a cuatro perspectivas:

1. La perspectiva del cliente con cinco indicadores, en la que los resultados personales son los indicadores.

2. La perspectiva del crecimiento organizacional, en la visión de futuro y de maduración de todas las organizaciones, con cinco indicadores más.

3. La perspectiva del análisis económico y en cómo invertimos; qué relación existe entre la inversión económica de la organización con el tiempo de apoyo dedicado a los usuarios; o la relación que el dinero tiene con los resultados que se obtienen, con cinco indicadores más.

4. La perspectiva sobre los procesos internos organizacionales con cuatro indicadores más.

Estas cuatro perspectivas, que están hechas en base a valorar los procesos de cambio, nos dan una primera visión de resultados de cambio y servirán a las organizaciones para autoevaluarse y para generar estrategias de cambio; y también pueden ser utilizadas en el ámbito de la política pública. De todas formas, la política pública también debe tener en cuenta otras variables que en este instrumento no se desarrollan, como son: variables de población, de situación social, etc., que deben tenerse en cuenta a la hora de establecer recursos o desarrollar planes.

Hay muchas más variables que afectan a un cambio organizacional y social que tienen que ver con la formación de los profesionales y con la utilización y aprovechamiento de los recursos.

P: ¿Qué opinión les merece la formación inicial de los profesionales y en particular de los maestros en el ámbito de la DID? o ¿Cuál sería el formato de formación (inicial y durante el ejercicio profesional) más adecuado en el ámbito de la DID?

DM: Respecto a la formación de los profesionales (maestros, otros) debo decir que, en principio, tenemos dos opciones a la hora de formar a los profesionales.

Una de ellas es la del "superespecialista”, siendo ejemplos de ello los que se dan en los sistemas educativos centroeuropeos (facultades de pedagogía terapéutica) como es el caso de Alemania y algunos países del Este.

La otra opción, de la que soy más partidario, es la de los modelos anglosajones, fundamentalmente el británico; en los cuales el profesor que se ocupa de este alumnado es, fundamentalmente, un profesor que sabe ligar su trabajo para con esta población con el currículum ordinario, y que también tiene la formación de base mínima para dar apoyo a las diferentes discapacidades. 
De todas formas, este tema, con la libertad que han tenido las Universidades dentro del Plan de Formación que se ha venido en llamar "Plan Bolonia", el sistema se ha desmadejado un poco, y echo a faltar una cierta reflexión de conjunto, acuerdos interuniversitarios, directrices de buenas prácticas, e incluso una presencia más activa de las entidades del sector en reclamar determinados elementos en el currículum de formación del profesorado.

Así pues, es una pregunta difícil de contestar, si no conocemos el Plan de Estudios de cada Universidad que está formando a profesores especializados en respuesta a las necesidades educativas especiales (Pedagogía Terapéutica).

CG: Frente a estas cuestiones, y coincidiendo con lo expuesto por Delfín, me gustaría poner de relieve el hecho que deberíamos distinguir entre aquellos profesionales que llevan a cabo un rol en un servicio en el campo de la DI, pero que pueden proceder de disciplinas distintas, como es el caso de la Atención Precoz o Temprana (en él conviven psicólogos, pedagogos, logopedas...) de otros en que su rol profesional en el servicio está asociado a una disciplina.

En cualquier caso, el modelo que me parece más defendible, el más potente, es el de una formación inicial rigurosa, y luego una especialización mediante un máster.

EL MODELO FORMATIVO DE LOS PROFESIONALES QUE TRABAJAN CON PERSONAS CON DID QUE PARECE MÁS DEFENDIBLE, EL MÁS POTENTE, ES EL DE UNA FORMACIÓN INICIAL RIGUROSA, Y LUEGO UNA ESPECIALIZACIÓN MEDIANTE UN MÁSTER

Con ello, no vengo a decir nada nuevo, pero la realidad es que, debido a diversas razones, ya sea por cuestiones presupuestarias u otras, no se está dando este enfoque de formación.

Pensando en los maestros y profesores de apoyo en el ámbito de la educación especial, no renuncio a decir que existe un problema de origen que deberíamos denunciar; y es el que se deriva de la normativa del Ministerio de Educación actual que iguala la mención con la especialización. Los Grados de Magisterio tienen 30 créditos de los estudios de Grado que se hacen equivalentes a la especialización; esto es un fraude. Luego resulta que cualquier persona, con una mínima formación, puede ocupar el papel que antes estaba reservado al especialista en educación especial. Así no se contribuye a una escuela inclusiva ni, por supuesto, a una educación de calidad.

El debate entre especialista y generalista es un debate importante; si hay un maestro generalista bien formado, y la especialización viene vehiculada a través de un máster, creo que estaríamos en mejores condiciones que las de ahora. 


\section{A modo de decálogo de conclusiones}

Finalmente, exponemos las diez conclusiones que nos han parecido más relevantes y que han sido validadas por los propios participantes del debate:

$\checkmark$ LA CONDUCTA ADAPTATIVA TIENE MÁS PESO QUE LA INTELIGENCIA PARA DISCRIMINAR EL DIAGNÓSTICO Y PARA ENTENDER UN POCO EL FUNCIONAMIENTO DE LA PERSONA

$\checkmark \quad$ ES PRECISO DISPONER DE UN MODELO MÁS DEFINIDO DE POLÍTICAS PÚBLICAS QUE TENGA EN CUENTA LOS RESULTADOS PERSONALES

$\checkmark$ CUANDO LOS SERVICIOS QUE LAS ORGANIZACIONES OFRECEN A LAS FAMILIAS ESTÁN MUY ENRAIZADOS EN EL MODELO DE EXPERTOS, SE LIMITA LA PARTICIPACIÓN DE LAS FAMILIAS, ASIGNÁNDOLES UN PAPEL DE MEROS RECEPTORES DE SUS ORIENTACIONES

$\checkmark$ LO QUE DEBE IMPORTAR A LAS ORGANIZACIONES Y A LAS ADMINISTRACIONES PÚBLICAS SON LOS RESULTADOS PERSONALES QUE PODAMOS OBTENER EN TÉRMINOS DE MEJORA DE CALIDAD DE VIDA

$\checkmark$ LA AUTODETERMINACIÓN ES UNA DE LAS DIMENSIONES DE CALIDAD DE VIDA EN LA QUE LAS PERSONAS SE MUESTRAN MÁS NECESITADAS

$\checkmark$ EL MODELO DE CALIDAD DE VIDA ESTÁ SIRVIENDO DE REVULSIVO Y PROMUEVE EL CAMBIO ORGANIZACIONAL

$\checkmark \quad$ LO QUE HACE LA CALIDAD DE VIDA ES DAR UNA GRAN IMPORTANCIA A LOS RESULTADOS PERSONALES

$\checkmark \quad$ LAS DIMENSIONES DE LA CALIDAD DE VIDA SON EL MEJOR SISTEMA PARA INTEGRAR LA INFORMACIÓN DIAGNÓSTICA, EVALUATIVA, DE APOYOS, DE DIFERENTES PROFESIONALES

$\checkmark$ EL FUTURO NOS VA A GENERAR INVESTIGACIÓN QUE INTENTARÁ ASOCIAR LOS RESULTADOS DEL DIAGNÓSTICO AL SISTEMA DE APOYOS

$\checkmark$ EN NUESTRA LEGISLACIÓN LA INCLUSIÓN ESTÁ MUY PRESENTE, PERO EN LA REALIDAD QUEDA TODAVÍA LEJOS

\section{Referencias bibliográficas}

Castelló, A. (2002). Delimitación conceptual de la inteligencia. Un análisis de las dimensiones física, funcional y conductual. Boletín de Psicología, 74, 7-26

Castelló, A. (2010). Intel-ligència al segle XXI. Perspectiva Escolar, 345, 2-12.

Castello, A. y Cano, M. (2011). Inteligencia interpersonal: conceptos clave. Revista Electrónica Interuniversitaria de Formación del Profesorado, vol. 14, 3.

Greenspan, S. (1979). Social intelligence in the retarded. En N. R. Ellis (Ed.), Handbook of mental deficiency, psychological theory and research, 2nd edition (pp. 483-531). Hillsdale, NJ: Erlbaum.

GreENSPAN, S. (1981). Social competence and handicapped individuals: Implications of a proposed model. En B. K. KeOgh (Ed.), Advances in Special Education, 3 (pp. 41-82). 
Greenspan, S. (1994). Review of the 1992 AAMR Definition, Classification, and Systems of Supports. American Journal on Mental Retardation, 98, 544-549.

GreENSPAN, S. I. (1997). The growth of the mind: And the endangered origins of intelligence. New York: Addison-Wesley

Greenspan, S. (1999). What is meant by mental retardation? International Review of Psychiatry, 11, 6-18.

GreENSPAN, S. (2006). Mental retardation in the real world: Why the AAMR definition is not there yet. En H. N. SwiTZKy y S. GreENSPAN (Eds.), What is MR: Ideas for an evolving disability (pp. 165-183). Washington, DC: American Association on Mental Retardation.

Greenspan, S. y Granfield, J. M. (1992). Reconsidering the construct of mental retardation: Implications of a model of social competence. American Journal of Mental Retardation, 96, 4, 442-453.

Greenspan, S., Switzsky, H. y Granfield, J. (1995). Everyday intelligence and adaptive behavior: A theoretical framework. En J. JACOBSON y J. Mulick (Eds.), Manual on diagnosis and professional practice in mental retardation. Washington, DC.: American Psychological Association.

Greenspan, S., Switzsky, H. y Woods, G. W. (2011). Intelligence involves risk-awareness and intellectual disability involves risk-unawareness: Implications of a theory of common sense. Journal on Intellectual and Developmental Disability, 36 (4), 246-257.

SCHALOCK, R. L. (1998a). A quest for quality: Achieving organizational outputs and personal outcomes. En J. GARDNER y S. Nudler (Eds.), Leadership, quality, and organizational dynamics. Baltimore: Brookes.

Schalock, R. L. (2001). Conducta adaptativa, competencia personal y calidad de vida. Siglo Cero: Revista Española sobre Discapacidad Intelectual, vol. 32, 194 17-28.

SCHALOCK, R. L. (2013). Integrando el concepto de calidad de vida y la información de la Escala de Intensidad de Apoyos en planes individuales de apoyo. Siglo Cero: Revista Española sobre Discapacidad Intelectual, vol. 44, 245, 6-21.

SHOGREN, K. A. (2011). Culture and self-determination: A review of the literature and directions for future research and practice. Career Development for Exceptional Individuals, 34, 115-127.

Shogren, K. A. (2012). Hispanic mothers' perceptions of self-determination. Research and Practice for Persons with Severe Disabilities, 37, 170-184.

SHOgRen, K. A. (2013). Considering context: An integrative concept for promoting outcomes in the intellectual disability field. Intellectual and Developmental Disabilities, 51, 132-137.

Shogren, K. A., Bradley, V., Gómez, S., Yeager, M. y Schalock, R. L. (2011). Política pública y mejora de los resultados deseados para las personas con discapacidad intelectual. Siglo Cero: Revista Española sobre Discapacidad Intelectual, vol. 42, 238, 7-25.

Shogren, K. A. y Broussard, R. (2011). Exploring self-advocates' perceptions of self-determination. Intellectual and Developmental Disabilities, 49, 86-102.

Shogren, K. A., Lang, R., Machalicek, W., Rispoli, M. y O’Reilly, M. (2011). Self- versus teacher-management of behavior for elementary school students with Asperger syndrome: Impact on classroom behavior. Journal of Positive Behavior Interventions, 13, 87-96.

Shogren, K. A., Palmer, S. B., Wehmeyer, M. L., Williams-Diehm, K. y Littile, T. D. (2012). Effect of intervention with the Self-Determined Learning Model of Instruction on access and goal attainment. Remedial and Special Education, 33, 320-330.

Shogren, K. A. y Plotner, A. J. (2012). Characteristics of transition planning for students with intellectual disability and autism: Data from the National Longitudinal Transition Study-2. Intellectual and Developmental Disabilities, 50, 16-30. 
Shogren, K. A., Wehmeyer, M. L., Palmer, S. B. y Paek, Y. (2013). Exploring personal and school environment characteristics that predict self-determination. Exceptionality, 21, 147-157.

Verdugo, M. Á. y Schalock, R. L. (2013). El cambio en las organizaciones de discapacidad. Madrid: Alianza Editorial.

WehmeYer, M. L. (Ed.) (2013). Handbook of positive psychology and disability. UK: Oxford University Press.

Wehmeyer, M. L. (Ed.) (2013). The story of intellectual disability: An evolution of meaning, understanding, and public perception. Baltimore: Paul H. Brookes.

Wehmeyer, M. L. (2013). Disability, disorder, and identity. Intellectual and Developmental Disabilities, 51 (2), 122-126.

Wehmeyer, M., Agran, M., Hughes, C., Martin, J., Mithaug, D. E. y Palmer, S. (2007). Promoting Self-Determination in Students with Developmental Disabilities. Guilford.

Wehmeyer, M. L. y Schalock, R. L. (2002). Autodeterminación y calidad de vida: implicaciones para los servicios de educación especial y para los apoyos. Siglo Cero: Revista Española sobre Discapacidad Intelectual, vol. 33, 201, 15-32.

Wehmeyer, M. L., Palmer, S., Shogren, K., Williams-Diehm, K. y Soukup, J. (2013). Establishing a causal relationship between interventions to promote self-determination and enhanced student self-determination. Journal of Special Education, 46 (4), 195-210. 